\title{
Structure-Induced Ultratransparency in Photonic Crystals
}

Jie Luo and Yun Lai

Additional information is available at the end of the chapter

http://dx.doi.org/10.5772/intechopen.71274

\begin{abstract}
This chapter presents the recent progress on structure-induced ultratransparency in both one- and two-dimensional photonic crystals (PhCs). Ultratransparent PhCs not only have the omnidirectional impedance matching with the background medium, but also have the ability of forming aberration-free virtual images. In certain frequency regimes, such ultratransparent PhCs are the most transparent solid materials on earth. The ultratransparency effect has many applications such as perfectly transparent lens, transformation optics (TO) devices, microwave transparent devices, solar cell packaging, etc. Here, we demonstrate that the ultratransparent PhCs with "shifted" elliptical equal frequency contour (EFC) not only provide a low-loss and feasible platform for transformation optics devices at optical frequencies, but also enable new degrees of freedoms for phase manipulation beyond the local medium framework. In addition, microwave transparent devices can be realized by using such ultratransparent PhCs.
\end{abstract}

Keywords: ultratransparency, photonic crystals, impedance matching, spatial dispersion, transformation optics

\section{Introduction}

Photonic crystals (PhCs), as periodic arrangement of dielectrics, affect the motion of photons and electromagnetic (EM) waves in much the same way that semiconductor crystals affect the propagation of electrons, providing a new mechanism to control and manipulate the flow of light at wavelength scale [1-5]. The key property of the PhCs is the photonic band gap induced from the periodic modulation of photons and EM waves, which can block wave propagation in certain or all directions. As photonic band-gap materials, PhCs play vital roles in light confinement and optical manipulation, promising many important applications, such as omnidirectional reflectors [6, 7], waveguides [8, 9], fibers [10], high-Q nanocavities and laser [11, 12], and angular filters [13]. 
However, little attention has been paid to the effect of enhancing transparency. Although there are pass bands in PhCs, they are usually reflective and, therefore, not transparent enough. As we know, transparent media are the foundation of almost all optical instruments, such as optical lens. However, perfect transparency has never been realized in natural transparent solid materials such as glass because of the impedance mismatch with free space. On the other hand, in the past decades, artificial EM materials like metamaterials [14-20] have been proposed to realize unusual EM properties beyond natural materials. However, most of the researches were focused on the realization of abnormal refractive behaviors such as negative refraction. Transparency over a large range of incident angle or a large frequency spectrum is theoretically possible, but the experimental realization is very difficult as complex and unusual parameters are required.

The photonic band-gap effect is actually induced by the periodic modulation of the reflections on the surfaces of dielectrics. That is, the periodic modulation strengthens the reflections on the surfaces of dielectrics to form a complete band gap at particular frequencies. Then, a natural question is: Is it possible to rearrange the periodic modulation of the reflections on the surfaces of dielectrics to make them cancel each other for all incident angles, so that omnidirectional impedance matching and omnidirectional perfect transmission can be realized?

In this chapter, we show the opposite effect of the band-gap effect in PhCs, i.e., the structureinduced ultratransparency effect [21, 22]. Ultratransparent PhCs not only have the omnidirectional impedance matching with the background medium, but also have the ability of forming aberration-free virtual images. The equal frequency contours (EFCs) of such ultratransparent PhCs are designed to be elliptical and "shifted" in the k-space and thus contain strong spatial dispersions and provide more possibilities for omnidirectional impedance matching. Interestingly, the combination of perfect transparency and elliptical EFCs satisfies the essential requirement of ideal transformation optics (TO) devices [23-27]. Therefore, such ultratransparent PhCs not only provide a low-loss and feasible platform for TO devices at optical frequencies, but also enable new freedom for phase manipulation beyond the local medium framework. Moreover, such ultratransparent PhCs have shown enormous potential applications in the designs of microwave transparent wall, nonreflection lens, omnidirectional polarizer, and so on.

\section{Ultratransparency effect: the opposite of the band-gap effect}

\subsection{Definition of ultratransparency: omnidirectional impedance matching and aberration- free virtual image}

It is well known that the band-gap effect induced by the periodic distribution of dielectrics can block the propagation of EM waves in certain or all directions, as illustrated in Figure 1(a). The forbiddance of wave propagation is the result of lacking propagation modes within the PhCs, which can be seen from the EFCs in Figure 1(a). The circle in the left denotes the EFC of free space, and there is no dispersion of the PhCs within the band gap. Interestingly, we would like to rearrange the periodic array of dielectrics to obtain the omnidirectional impedance matching effect, which allows near 100\% transmission of light at all incident angles, as illustrated in Figure 1(b). 
(a)
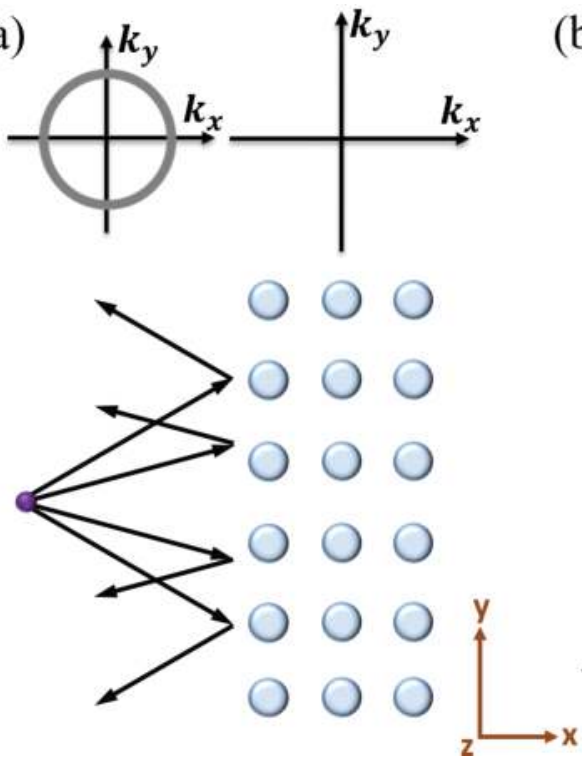

(b)
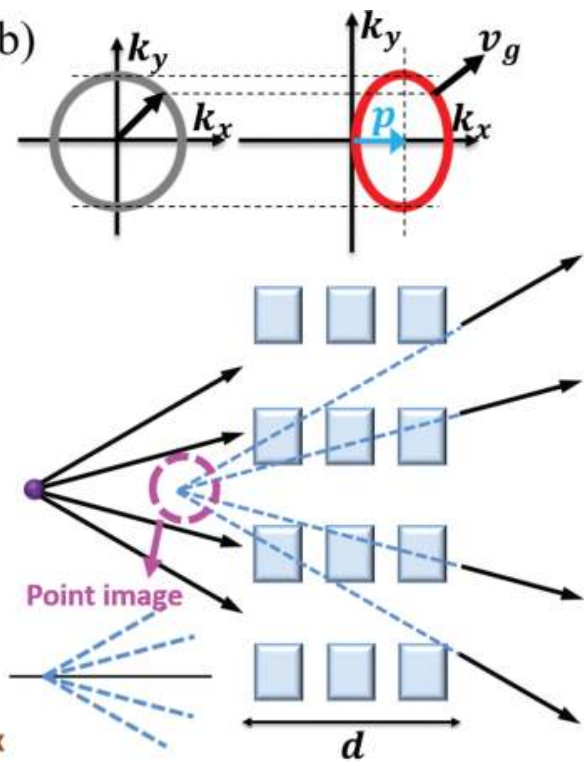

Figure 1. (a) Total reflection by a PhC slab with complete photonic band gap. (b) Aberration-free virtual image formation through an ultratransparent medium without any reflection due to omnidirectional impedance matching. The arrows and dashed lines in (a) and (b) represent the light rays from a point source, and the back-tracing lines, respectively. The inset graphs show the corresponding EFCs.

Moreover, we expect such media to have the ability of forming aberration-free virtual images, which is absent in normal transparent media like glass. By using ray optics, it can be easily shown that transmitted rays from a point source behind a dielectric slab would form a "blurred" area of virtual image rather than a point image. For demonstration, we placed a point source on the left side of a glass slab (with a thickness of $d$ and a refractive index of 1.46) at a distance of $d$, as shown in Figure 2(a). The virtual image is formed by the back tracing lines (thin lines) of the transmitted waves in the right side (thick lines), showing evident aberrations. In Figure 2(b), we present the zoom in "blurred" image in the region marked by dashed lines in Figure 2(a), showing the spatial distribution of the formed virtual image. This implies that the position of the virtual image will change if we observe from different positions, as illustrated in Figure 2(c).

Actually, such aberrations of the virtual image originate from the mismatch between the EFCs of free space and dielectrics, i.e., their EFCs do not have the same height in the transverse direction (i.e., the $k_{y}$ direction in Figure 1) [21]. By using ray optics, it can be demonstrated that a circle or elliptical EFC having the same height of the EFC of free space in the $k_{y}$ direction enables the formation of aberration-free virtual images [21], i.e., the EFC has the form of

$$
\frac{\left(k_{x}-p\right)^{2}}{q}+k_{y}^{2}=k_{0}^{2}
$$



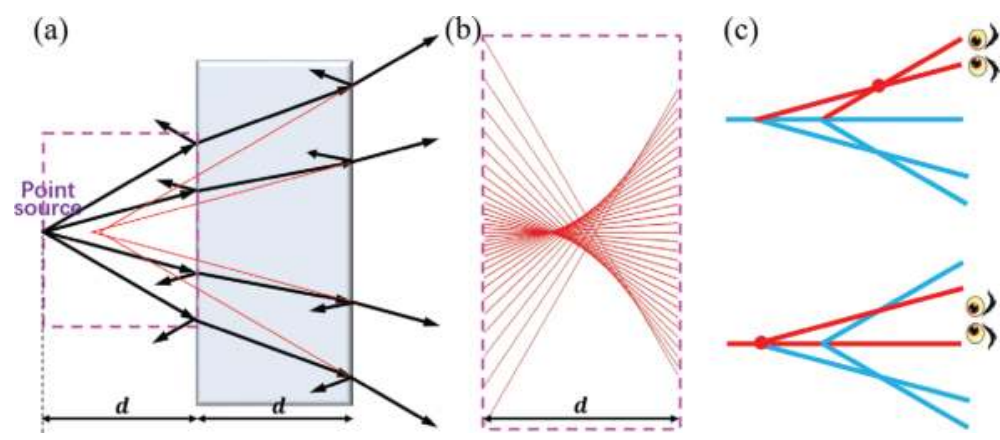

Figure 2. The formation of "blurred" virtual image by a glass slab. (a) The existence of general reflection and aberration in virtual images behind a glass slab. (b) The zoom in "blurred" image in the region marked by dashed lines in (a). (c) The position of the virtual image for the observation from different positions.

where $k_{0}$ is the wave number in air, $k_{x(y)}$ is the $x(y)$ component of the wave vector, $p$ denotes the displacement from the Brillouin zone center, and $q$ determines the ratio of the $k_{y}$ and $k_{x}$ axes of the ellipse, as shown in Figure 1(b). We note that the "shift" of $p$ in the $k_{x}$ direction does not affect the formation of virtual images, because such a "shift" does not change the refractive behavior at all in this case.

Supposing that the rearrangement of the periodic array of dielectrics not only makes the whole structure impedance matched to free space for all incident angles, but also creates the unique EFC described by Eq. (1); thus, both omnidirectional $100 \%$ perfect transmission and aberration-free virtual imaging are enabled simultaneously. Apparently, such a level of transparency is superior to that of normal transparent media like dielectrics and is thus hereby denoted as ultratransparency.

\subsection{Ultratransparency based on local and nonlocal media}

According to Fresnel equations, reflection of light on the surface of dielectrics is inevitable, except at a single-incident angle referred to as the Brewster angle under transverse magnetic (TM) polarization, as demonstrated in Figure 3(a). Here, we extend the impedance matching from one particular angle (i.e., Brewster angle) to all incident angles in a nonlocal or spatial dispersive medium, whose effective permittivity $\varepsilon\left(k_{y}\right)$ and permeability $\boldsymbol{\mu}\left(k_{y}\right)$ are dependent on wave vectors.

To begin with, we assume that the nonlocal medium exhibits an irregular-shaped EFC shown in Figure 3(b). An incident wave of transverse electric (TE) polarization with electric fields polarized in the $z$ direction is considered. For the TE polarization, the dispersion relation of the nonlocal medium can be expressed in a general form as,

$$
\frac{k_{x}^{2}}{\mu_{y}\left(k_{y}\right)}+\frac{k_{y}^{2}}{\mu_{x}\left(k_{y}\right)}=\varepsilon_{z}\left(k_{y}\right) k_{0}^{2},
$$

which determines the relationship between the components of wave vectors $k_{x}$ and $k_{y}$. 

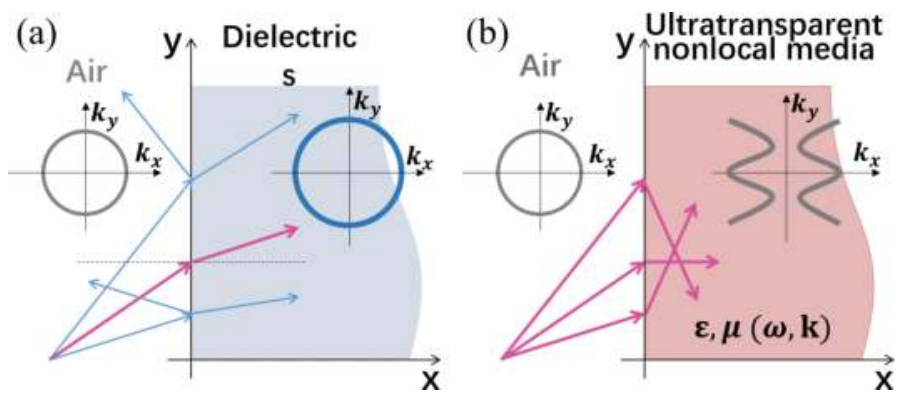

Figure 3. (a) There exist reflected waves at the interface of free space and dielectrics except for the Brewster angle for TM polarization. (b) Nonreflection and total transmission on the surface of ultratransparent nonlocal media for all incident angles. The thin circle, thick circle, and curves in the inset graphs denote the EFCs of air, dielectrics, and the nonlocal media, respectively.

In this case, the wave impedance of free space can be derived as $Z_{0} \equiv \frac{E_{z}}{H_{y}}=-\frac{\mu_{0}}{k_{x, 0}} \omega$ for TE polarization [28-30]. $E_{z}$ and $H_{y}$ are the $z$-component of electric fields and the $y$-component of magnetic fields, respectively. $k_{x, 0}, \mu_{0}$, and $\omega$ are the $x$-component of wave vector in free space, the permeability of vacuum, and angular frequency of the EM wave, respectively. Similarly, the wave impedance of the nonlocal medium can be written as $Z=-\frac{\mu_{0} \mu_{y}\left(k_{y}\right)}{k_{x}} \omega$. Then, the impedance matching of the nonlocal medium and free space requires $Z=Z_{0}$. By considering dispersion relations, the impedance matching condition is obtained as,

$$
\frac{\mu_{x}\left(k_{y}\right) \mu_{y}\left(k_{y}\right)}{\mu_{x}\left(k_{y}\right) \varepsilon_{z}\left(k_{y}\right) k_{0}^{2}-k_{y}^{2}}=\frac{1}{k_{0}^{2}-k_{y}^{2}}
$$

If Eq. (3) can be satisfied for all $\left|k_{y}\right|<k_{0}$, then omnidirectional impedance matching can be achieved, leading to near $100 \%$ transmission of light for all incident angles.

An obvious local medium solution of Eq. (3) is that $\mu_{x} \mu_{y}=1$ and $\mu_{x} \varepsilon_{z}=1$, which correspond to elliptical EFCs centered at the Brillouin zone center, are consistent with transformation optics theory [23-27]. However, this is not the only possible solution for spatially dispersive media. Now, we consider medium with the EFC described by Eq. (1), i.e., $k_{x}=p \pm \sqrt{q\left(k_{0}^{2}-k_{y}^{2}\right)}$. By substituting this relation into Eq. (3) and the spatial dispersion, we obtain the analytical forms of spatially dispersive parameters as:

$$
\mu_{y}^{2}\left(k_{y}\right)=\frac{\left[p \pm \sqrt{q\left(k_{0}^{2}-k_{y}^{2}\right)}\right]^{2}}{k_{0}^{2}-k_{y}^{2}} \text { and } \varepsilon_{z}\left(k_{y}\right) k_{0}^{2}-\frac{k_{y}^{2}}{\mu_{x}\left(k_{y}\right)}= \pm\left[p \pm \sqrt{q\left(k_{0}^{2}-k_{y}^{2}\right)}\right] \sqrt{k_{0}^{2}-k_{y}^{2}}
$$

Eq. (4) shows that when EM parameters possess spatial dispersions, there exist infinite solutions of $\mu_{y}\left(k_{y}\right), \varepsilon_{z}\left(k_{y}\right)$, and $\mu_{x}\left(k_{y}\right)$ for the satisfaction of Eq. (4) for all $\left|k_{y}\right|<k_{0}$, indicating infinite solutions for omnidirectional impedance matching. 


\section{Structure-induced ultratransparency in two-dimensional PhCs}

\subsection{Nonlocal effective medium theory}

For the design of ultratransparent PhCs, we first propose a nonlocal effective medium theory for the homogenization of PhCs. Here, we consider uniform plane wave incidence. In this situation, the validity of the nonlocal effective medium theory lies in the satisfaction of the following four premises: (1) single-mode approximation [28], i.e., only one eigen-mode is excited; (2) the amplitudes of fields at the incident boundary are almost constant; (3) the phases of fields at the incident boundary obey the trigonometric functions; and (4) the electric and magnetic fields are in phase at the incident boundary. Although these premises are seemingly stringent, it turns out that most eigen-modes of the first few bands (e.g., monopolar and dipolar bands) can indeed satisfy these requirements (Figure 4).

With the assumption of the abovementioned premises, eigen-fields of TE polarization at the boundary $x=0$ can be approximately expressed as $\mathbf{F}=\mathbf{F}_{0} e^{i\left(k_{x} x+k_{y} y\right)} e^{-i \omega t}$ with $\mathbf{F}_{0}=E_{0} \widehat{z}, H_{0} \widehat{y}$. $E_{0}$ and $H_{0}$ are the amplitudes of eigen-electric and eigen-magnetic fields, respectively, which are almost independent of $y$. This indicates that the eigen-fields are the boundary and have the same formula as those in a uniform medium. Thus, the surface impedance of the PhCs can be calculated in a similar formula as [28-30],

$$
Z_{x}=\frac{\left\langle E_{z}\right\rangle_{x=0}}{\left\langle H_{y}\right\rangle_{x=0}} \text { and } Z_{y}=\frac{\left\langle E_{z}\right\rangle_{x=0}}{\left\langle H_{x}\right\rangle_{x=0}}
$$

where $\langle F\rangle_{x=0}$ denotes the average of eigen-field $F$ along the unit cell boundary $x=0 . E_{z}, H_{x}$ and $H_{y}$ are the $z$-component of eigen-electric fields, $x$ - and $y$-components of eigen-magnetic fields, respectively. Based on Eq. (5), the surface impedance of a PhC can be calculated as long as the eigen-fields of the $\mathrm{PhC}$ are solved.

On the other hand, the PhC satisfying previous premises generally can be described as a

uniform medium with effective relative permittivity $\left(\begin{array}{lll}\varepsilon_{x, \text { eff }} & & \\ & \varepsilon_{y, \text { eff }} & \\ & & \varepsilon_{z, \text { eff }}\end{array}\right)$, relative

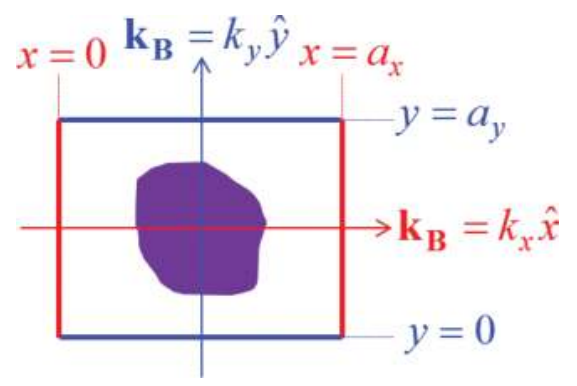

Figure 4. Schematic graph of the unit cell for the nonlocal effective medium theory. 
permeability $\left(\begin{array}{ccc}\mu_{x, \text { eff }} & & \\ & \mu_{y, \text { eff }} & \\ & & \mu_{z, \text { eff }}\end{array}\right)$, and dispersion $\frac{k_{x}^{2}}{\mu_{y, \text { eff }}}+\frac{k_{y}^{2}}{\mu_{x, \text { eff }}}=\varepsilon_{z, \text { eff }} k_{0}^{2}$. Thus, the impedance of the PhC can be rewritten as the function of effective parameters:

$$
Z_{x}=-\frac{\mu_{0} \mu_{y, e f f}}{k_{x}} \omega \text { and } Z_{y}=\frac{\mu_{0} \mu_{x, \text { eff }}}{k_{y}} \omega
$$

Compared with Eqs. (5) and (6), the effective parameters can be derived as,

$$
\begin{aligned}
& \varepsilon_{z, \text { eff }}=\frac{1}{\varepsilon_{0} \omega} \frac{k_{y}\left\langle H_{x}\right\rangle_{x=0}-k_{x}\left\langle H_{y}\right\rangle_{x=0}}{\left\langle E_{z}\right\rangle_{x=0}}, \\
& \mu_{x, \text { eff }}=\frac{k_{y}}{\mu_{0} \omega} \frac{\left\langle E_{z}\right\rangle_{x=0}}{\left\langle H_{x}\right\rangle_{x=0}} \text { and } \mu_{y, \text { eff }}=-\frac{k_{x}}{\mu_{0} \omega} \frac{\left\langle E_{z}\right\rangle_{x=0}}{\left\langle H_{y}\right\rangle_{x=0}}
\end{aligned}
$$

With Eq. (7), the effective parameters of the PhC can be obtained by analyzing the eigen-fields with Bloch wave vector $\mathbf{k}_{\mathbf{B}}=k_{x} \widehat{x}+k_{y} \hat{y}$. We note that the Bloch wave vector is restricted in the first Brillouin zone, and the choice of the eigen-fields of $-\mathbf{k}_{\mathbf{B}}$ or $+\mathbf{k}_{\mathbf{B}}$ depends on the direction of group velocity. In addition, we find that this method is still valid even for the eigen-modes far away from the Brillouin center, but the effective parameters are generally k-dependent, i.e., nonlocal or spatially dispersive.

\subsection{Two-dimensional ultratransparent PhCs}

PhCs contain strong spatial dispersions and thus provide the perfect candidate for realization of ultratransparency effect. Here, we demonstrate a type of PhCs composed of a rectangular array of dielectric rods in free space, with the unit cell shown in Figure 5(a). Under TE polarization, the band structure is presented in Figure 5(b), and the EFC of the third band is plotted in the reduced first Brillouin zone in Figure 5(c). The working frequency is chosen as $f a / c=0.3183$, where $f, a$, and $c$ are the frequency, the lattice constant, and the speed of light in free space, respectively. We see that the corresponding EFC (the right dashed lines in Figure 5(c)) is indeed a "shifted" ellipse that can be described by Eq. (1) with $p \approx \pi / a$. And the dispersion can be approximately written as,

$$
\left(\frac{k_{x}-\pi / a_{1}}{0.4541 a_{2} / 0.5097 a_{1}}\right)^{2}+k_{y}^{2}=\left(\frac{0.5097 \pi}{a_{2}}\right)^{2}
$$

Figure 5(d) shows the impedance difference of the $\mathrm{PhC}$ and free space of the third band, i.e., $\left|\frac{Z-Z_{0}}{Z+Z_{0}}\right|$, where $Z$ is the impedance of the PhC obtained from Eq. (5) and $Z_{0}$ is the impedance of free space. From Figure 5(d), it is seen that the impedance difference is very small for a very large range of $k_{y}$ at the working frequency, indicating impedance matching in a large range of incidence angles.

Moreover, in Figure 6(a), we present the effective parameters $\varepsilon_{z, \text { eff }}$ (square dots), $\mu_{x, \text { eff }}$ (circular dots), and $\mu_{y, \text { eff }}$ (triangular dots) of the PhC based on Eq. (7), showing the k-dependence. 
(a)
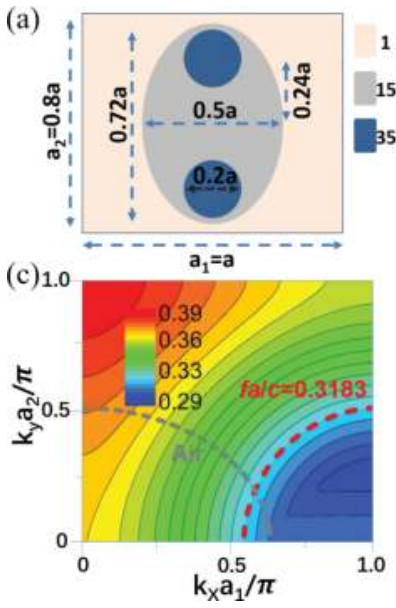

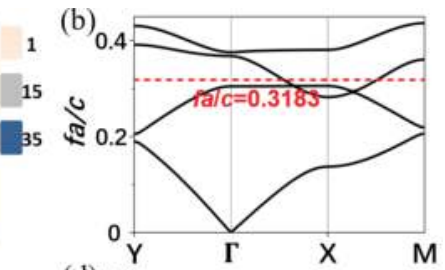

(d) 1.0

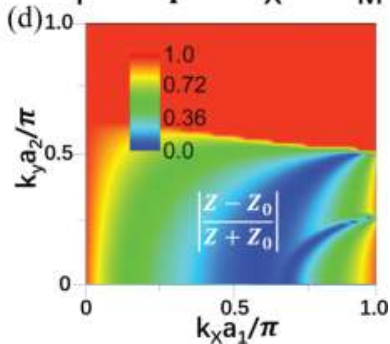

Figure 5. (a) Illustration of the unit cell of the ultratransparent PhC. (b) The band structure of the PhC. The dashed line denotes the working frequency $f a / c=0.3183$. (c) The EFC of the third band. The left and right dashed lines denote the EFCs of air and the PhC at the frequency $f a / c=0.3183$. (d) The impedance difference of the PhC and free space of the third band. Reprinted (figure) with permission from Ref. [21]. Copyright (2016) by the American Physical Society.

Interestingly, we find $\mu_{x, \text { eff }} \approx 1$, while $\varepsilon_{z, \text { eff }}$ and $\mu_{y, \text { eff }}$ are both dependent on $k_{y}$. The result of $\mu_{x, \text { eff }} \approx 1$ can be physically understood. Since the resonances are localized in the dielectrics and far away from the incident boundary, the modes propagating in the $y$ direction are weakly modulated by the periodicity. As a consequence, $\mu_{x, \text { eff }}$ has the same value as the relative permeability of the background medium.

Now, by substituting the condition $\mu_{x, \text { eff }} \approx 1$ and the dispersion Eq. (8) into Eq. (4), the analytical form of the effective parameters $\varepsilon_{z, \text { eff }}$ and $\mu_{y, \text { eff }}$ can be solved as,

$$
\begin{aligned}
& \varepsilon_{z, \text { eff }}\left(k_{y}\right)= \pm \frac{\pi}{a_{1} k_{0}^{2}}\left[1-0.4541 \sqrt{1-\left(\frac{k_{y} a_{2} / \pi}{0.5097}\right)^{2}}\right] \sqrt{k_{0}^{2}-k_{y}^{2}}+\frac{k_{y}^{2}}{k_{0}^{2}} \\
& \text { and } \mu_{y, \text { eff }}\left(k_{y}\right)= \pm \frac{\pi}{a_{1}}\left[1-0.4541 \sqrt{1-\left(\frac{k_{y} a_{2} / \pi}{0.5097}\right)^{2}}\right] / \sqrt{k_{0}^{2}-k_{y}^{2}}
\end{aligned}
$$

The choice of $\pm k_{x}$ depends on the direction of group velocity. Here, we choose $-k_{x}$, because hereby the band branch in the region $-\pi / a_{1}<k_{x}<0$ in the first Brillouin zone is excited, as determined by the direction of group velocity. In Figure 6(a), $\varepsilon_{z, \text { eff }}$ and $\mu_{y, \text { eff }}$ based on Eq. (9) (dashed lines) show perfect match with those obtained from Eq. (7) for all $k_{y}<k_{0}$, demonstrating the omnidirectional impedance matching effect.

For further verification, the transmittance through such a PhC slab consisting of $N(=4,5,6,15)$ layers of unit cells in the $x$ direction is numerically calculated, as presented in Figure 6 (b). It is shown that the transmittance is near unity $(>99 \%)$ for nearly all incident angles $\left(\theta<89^{\circ}\right)$ and is 

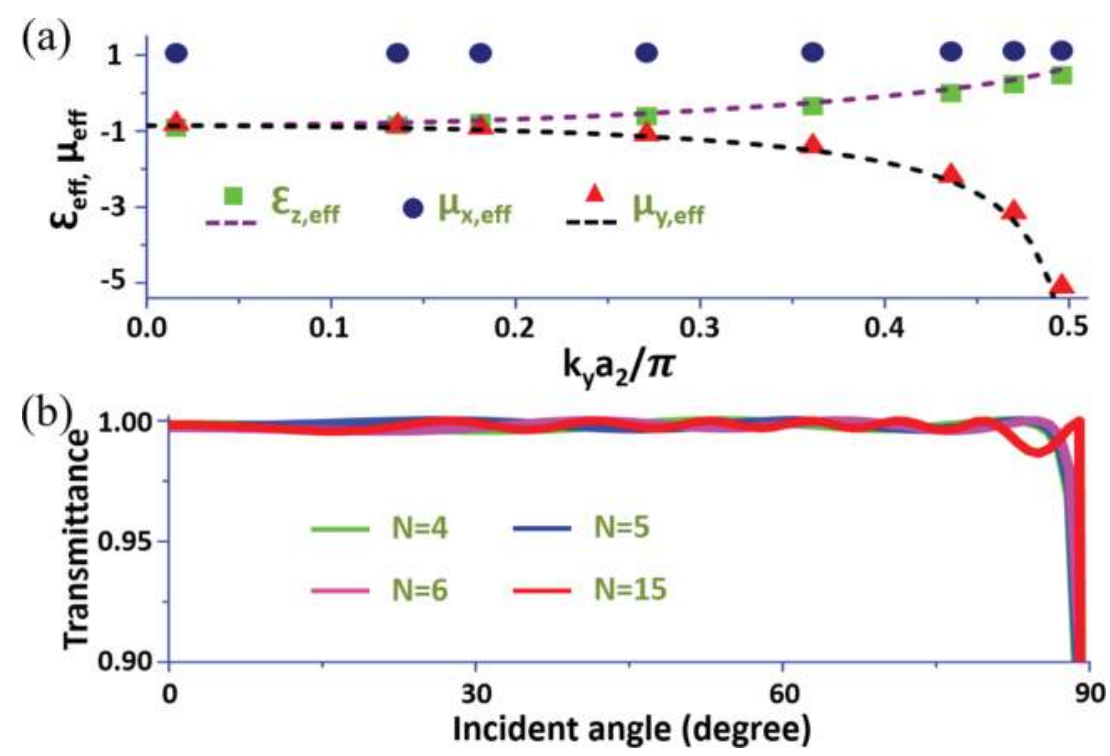

Figure 6. (a) The effective parameters $\varepsilon_{z, \text { eff }}, \mu_{x, \text { eff }}$, and $\mu_{y, \text { eff }}$ retrieved from the eigen-fields based on Eq. (7) (symbols) and predicted by the ultratransparency condition based on Eq. (9) (dashed lines). (b) Calculated transmittance through a PhC slab with $N(=4,5,6,15)$ layers of unit cells as the function of incident angles. Reprinted (figure) with permission from Ref. [21]. Copyright (2016) by the American Physical Society.

almost irrespective of the layer number $N$, indicating that the total transmission is a result of impedance matching instead of tunneling effects.

\subsection{Microwave experimental verification}

In fact, for transparency in a relatively smaller range of incident angles, the design process is much easier and the effect can exist in much simpler structures. In the following, we demonstrate a simple ultratransparent $\mathrm{PhC}$, which is verified by proof-of-principle microwave experiments. The PhC consists of rectangular alumina $(\varepsilon=8.5)$ bars in a square lattice, as illustrated by the inset in Figure 7(a). The lattice constant is set to be $a=12 \mathrm{~mm}$, and the length $\left(W_{1}\right)$ and width $\left(W_{2}\right)$ are $9.6 \mathrm{~mm}$ and $4.8 \mathrm{~mm}$, respectively, corresponding to $0.8 \mathrm{a}$ and $0.4 \mathrm{a}$. We consider TE polarization with electric fields polarized along the $z$ direction, and the band structure is presented in Figure 7(a). The normalized frequency is chosen to be $f a / c=0.4723$ (marked by a dashed line), corresponding to a working frequency around 11.8 GHz. In Figure 7(b), we can see that the shape of EFC of $f a / c=0.4723$ is nearly a part of "shifted" ellipse. And the dispersion can be approximately written as,

$$
\left(\frac{k_{x}-\pi / a_{1}}{0.4541 a_{2} / 0.5097 a_{1}}\right)^{2}+k_{y}^{2}=\left(\frac{0.5097 \pi}{a_{2}}\right)^{2}
$$

In addition, the impedance difference between the $\mathrm{PhC}$ and the free space is calculated by using Eq. (5), as shown in Figure 7(c). Clearly, the impedance difference is very small on the 
EFC of $f a / c=0.4723$ for a large range of $k_{y}$, demonstrating the wide-angle impedance matching effect.

Moreover, the effective parameters obtained from Eq. (7) are presented in Figure 8(a) by solid lines with symbols, showing $\mu_{x, \text { eff }} \approx 1$ for $k_{y}<0.818 \pi / a$ as the result of weakly modulated
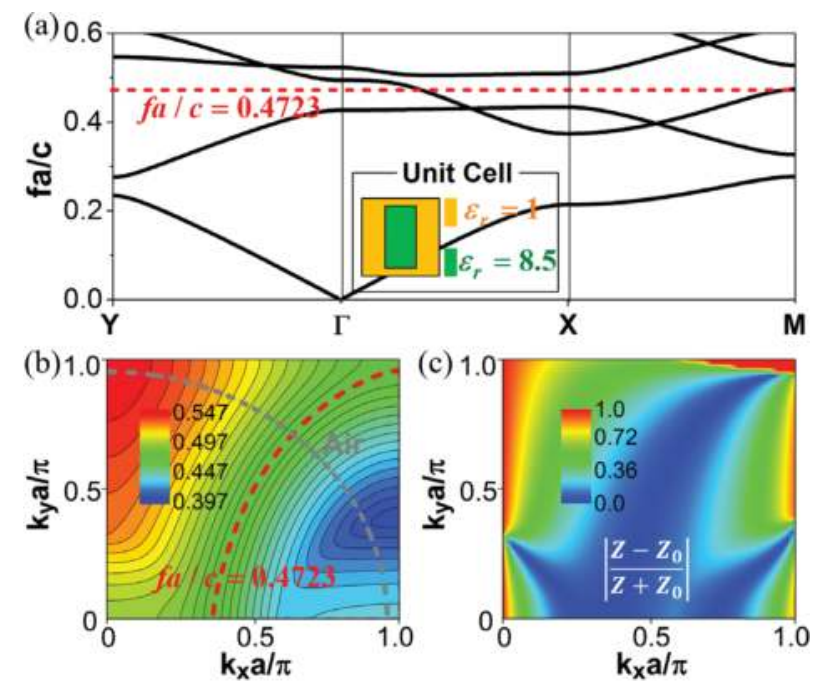

Figure 7. (a) Band diagram of the experimental $\mathrm{PhC}$ for TE polarization. The dashed line denotes the working frequency $f a / c=0.4723$. The inset is the illustration of the unit cell of the PhC. (b) EFCs in the frequency range $0.397 \leq f a / c \leq 0.547$. The left and right dashed lines denote the EFCs of air and the PhC at the frequency $f a / c=0.4723$. (c) The impedance difference of the $\mathrm{PhC}$ and the free space in the frequency range $0.397 \leq f a / c \leq 0.547$.

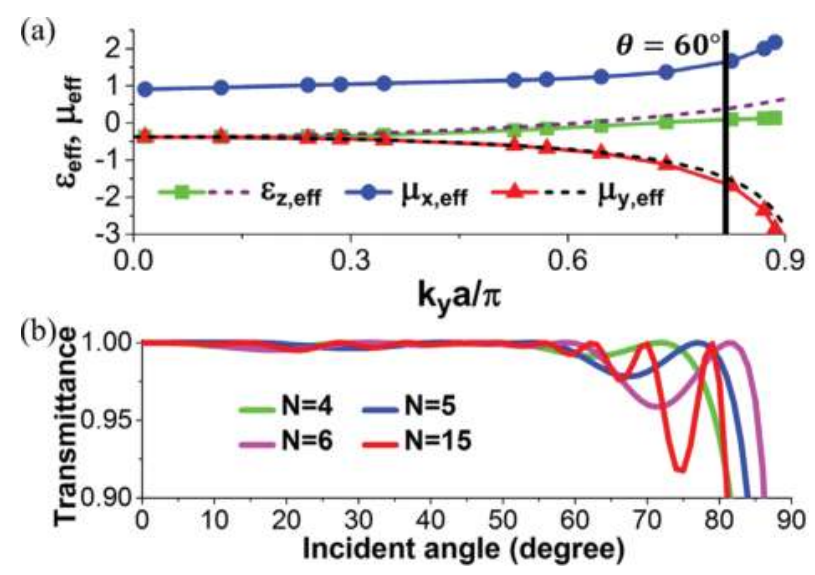

Figure 8. (a) Effective parameters $\varepsilon_{z, \text { eff }}, \mu_{x, \text { eff }}$, and $\mu_{y, \text { eff }}$ retrieved from the eigen-fields based on Eq. (7) (solid lines with symbols) and predicted by the ultratransparency condition based on Eq. (11) (dashed lines). (b) Transmittance through a $N(=4,5,6,15)$-layered PhC slab as the function of incident angles. 
modes propagating in the $y$ direction. By inserting the condition $\mu_{x, \text { eff }} \approx 1$ and the dispersion Eq. (10) into Eq. (4), the analytical expressions of $\varepsilon_{z, \text { eff }}$ and $\mu_{y, \text { eff }}$ can be derived as,

$$
\left\{\begin{array}{l}
\varepsilon_{z, \text { eff }}\left(k_{y}\right)= \pm \frac{\pi}{a k_{0}^{2}}\left[1.25-0.8945 \sqrt{1-\left(\frac{k_{y} a / \pi}{1.0415}\right)^{2}}\right] \sqrt{k_{0}^{2}-k_{y}^{2}}+\frac{k_{y}^{2}}{k_{0}^{2}} \\
\mu_{y . \text { eff }}\left(k_{y}\right)= \pm \frac{\pi}{a}\left[1.25-0.8945 \sqrt{1-\left(\frac{k_{y} a / \pi}{1.0415}\right)^{2}}\right] / \sqrt{k_{0}^{2}-k_{y}^{2}}
\end{array}\right.
$$

In Figure 8(a), the dashed lines denote $\varepsilon_{z, \text { eff }}$ and $\mu_{y, \text { eff }}$ based on Eq. (11) (negative sign is chosen), showing excellent coincidence with those from Eq. (7) for $k_{y}<0.818 \pi / a$ (i.e., $\theta<60^{\circ}$ ).

Furthermore, we calculate the transmittance through the PhC slab with $N(=4,5,6,15)$ unit cells, as shown in Figure 8(b). It is clearly seen that there exists near-unity transmittance for all
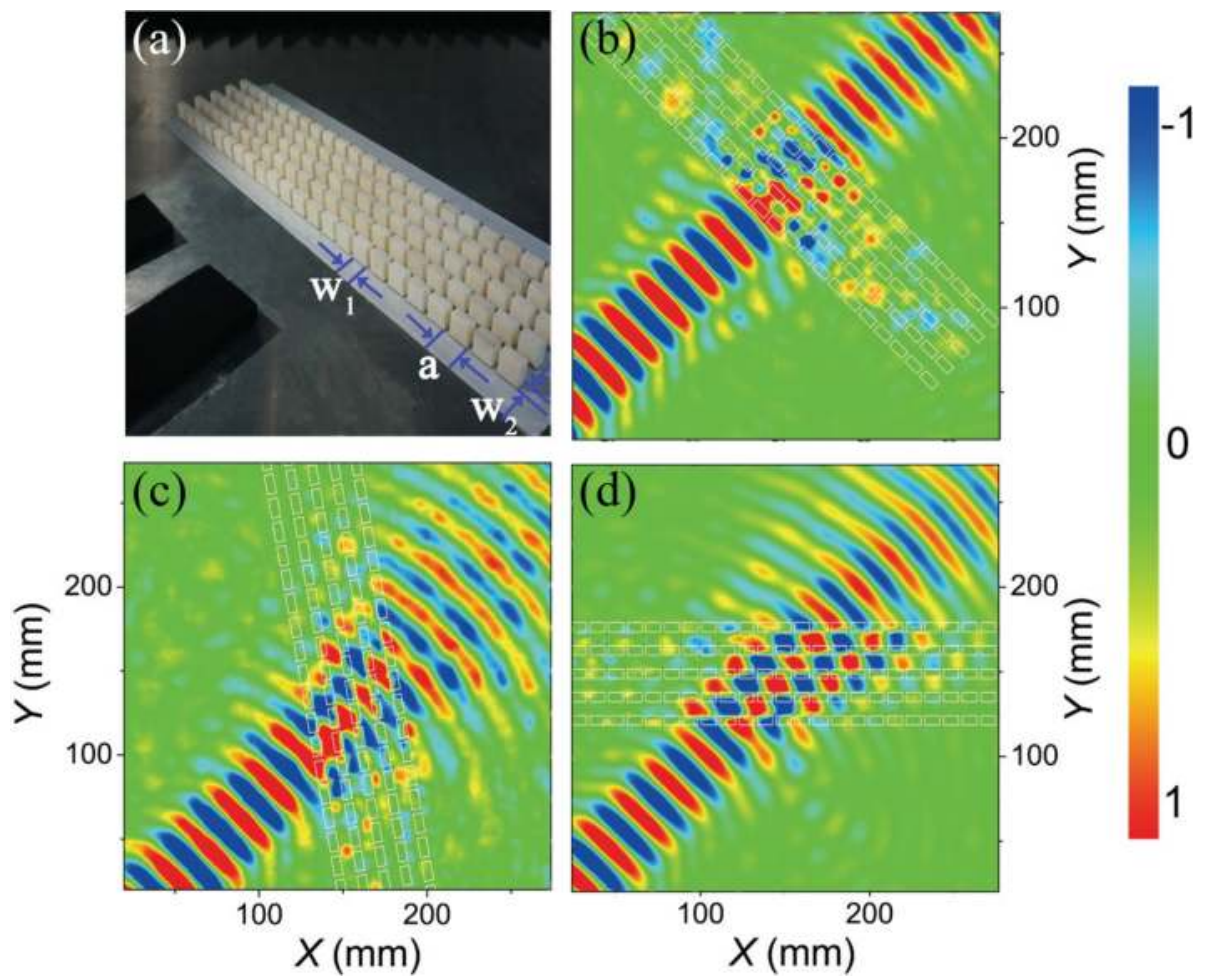

Figure 9. (a) Photo of the PhC composed of alumina bars (white) placed inside the microwave field mapper. The measured electric-field distributions for microwave beams with incident angles of (b) $0^{\circ}$, (c) $30^{\circ}$, and (d) $45^{\circ}$. Reprinted (figure) with permission from Ref. [21]. Copyright (2016) by the American Physical Society. 
incident angles of $\theta<60^{\circ}$ (i.e., $k_{y}<0.818 \pi / a$ ) irrespective of $N$, demonstrating the wide-angle impedance matching effect.

Next, we show microwave experimental results to verify the above theory. A $23 \times 5$ array of such a $\mathrm{PhC}$ is assembled in the $x y$ plane inside a parallel-plate waveguide composed of two flat aluminum plates, as shown in Figure 9(a). The separation between the two aluminum plates is $10.5 \mathrm{~mm}$, slightly larger than the height of the bars $(10 \mathrm{~mm})$, but smaller than half of the wavelength of interest $(25.4 \mathrm{~mm}$ for $11.8 \mathrm{GHz})$ to make sure that the whole experimental chamber can only support transverse EM modes. A microwave beam with a finite width was launched through a waveguide made of absorbing materials (in black in Figure 9(a)). The lower metal plate along with the $\mathrm{PhC}$ array was mounted on a translational stage. The electric field was measured via an antenna fixed in a hole in the upper metal plate (not shown here). Both the emitting and probing antennas are connected to our Agilent E5071C network analyzer to acquire the transmitted magnitude and phase of microwave signals. Such a setup allows us to measure the spatial distributions in the $x y$ plane for a series of incident angles from $0^{\circ}$ to $60^{\circ}$ at the working frequency of $11.8 \mathrm{GHz}$.

The measured electric fields for $0^{\circ}, 30^{\circ}$, and $45^{\circ}$ incident angles are displayed in Figure 9(b), (c), and (d), respectively. Clearly, the reflection is barely noticeable, indicating impedance matching for all these incident angles. In Figure 10, the measured transmittance (triangular dots) coincides with simulation results (solid lines) quite well, both showing great enhancement compared with that through an alumina slab with the same thickness (dashed lines). Although the ultratransparency effect is hereby only verified at the microwave frequency regime, the principle can be extended to optical frequency regime by using PhCs composed of silicon or other dielectrics.

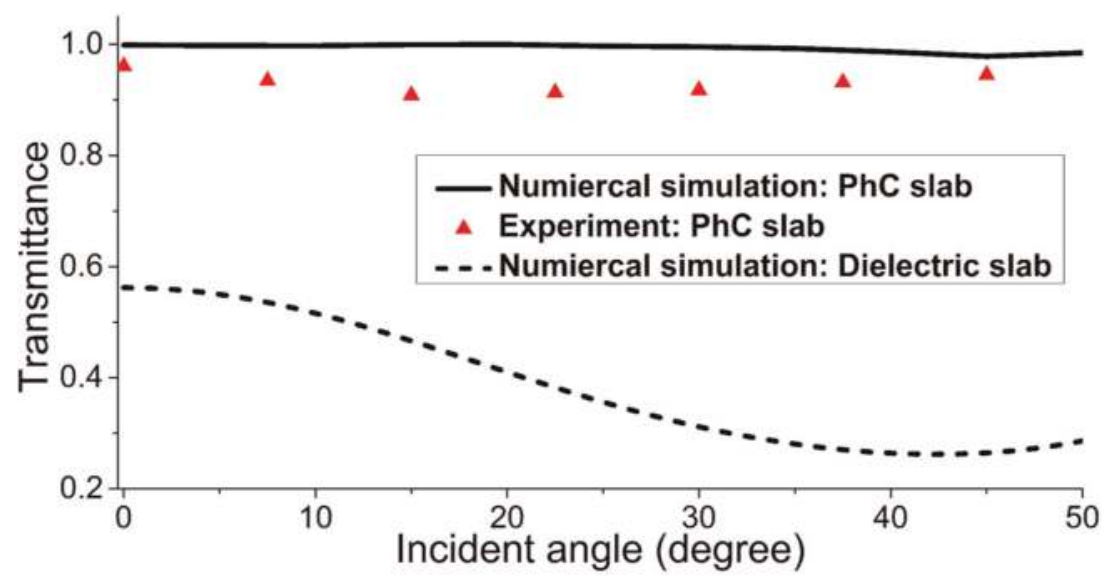

Figure 10. Transmittance through the PhC slab in simulations (solid lines) and experiments (triangular dots) and an alumina slab having the same thickness (dashed lines) as the function of incident angles. Reprinted (figure) with permission from Ref. [21]. Copyright (2016) by the American Physical Society. 


\section{Structure-induced ultratransparency in one-dimensional PhCs}

In the above, we have shown the structure-induced ultratransparency in two-dimensional PhCs. In the following, we demonstrate the structure-induced ultratransparency in onedimensional PhCs [22].

The one-dimensional ultratransparent $\mathrm{PhC}$ we studied is composed of two dielectric materials A and B stacked along the $x$ direction (Figure 11(c)). As we know, neither the dielectric material A nor B is perfectly transparent due to impedance mismatch. As a result, a large amount of EM waves are always reflected at the surface of dielectrics, as illustrated in Figure 11 (a) and (b). Interestingly, when we periodically stack the two dielectric slabs with appropriate filling ratio and lattice constant, the reflection waves on the dielectrics can cancel each other; thus, wide-angle and even omnidirectional nonreflection can be obtained, as illustrated in Figure 11(c).

Figure 12(a) presents the band structure of the $\mathrm{PhC}$, whose unit cell is constructed in a symmetric form, i.e., ABA structure, as shown by the inset in Figure 12(a). The relative permittivity and filling ratio of the material A (B) are 2 (6) and $0.6(0.4)$, respectively. The dashed line denotes the normalized frequency $f a / c=0.397$, which is chosen as the working frequency.

In Figure 12(b) and (d), the EFCs at the frequency $f a / c=0.397$ for the TE and TM polarizations are plotted, respectively. It is seen that the EFCs can be approximately regarded as a part of an ellipse with the center located at the $X$ point. In addition, the impedance difference between the PhC and air is shown in Figure 12(c) (for the TE polarization) and Figure 12(e) (for the TM polarization). It is seen that the impedance difference is very small for a large range of $k_{y}$ in the
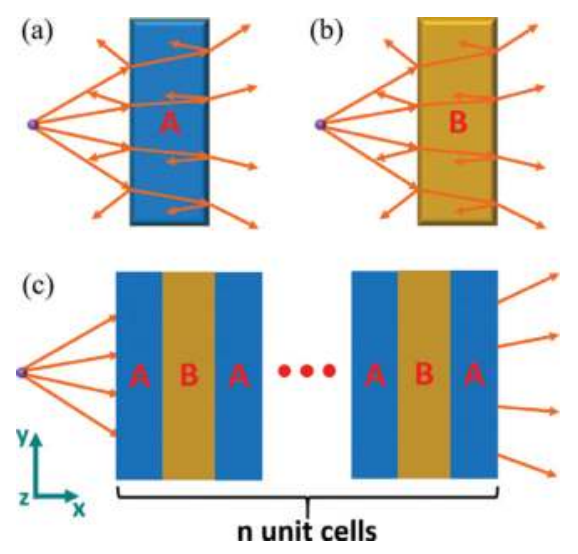

Figure 11. (a) and (b) show general reflection on the slab made of materials A and B, respectively. (c) Elimination of general reflection by a one-dimensional ultratransparent $\mathrm{PhC}$ composed of $\mathrm{ABA}$ unit cells. The orange arrows represent the rays of light emitted by a point source. Reprinted (figure) with permission from Ref. [22]. Copyright (2016) by the Optical Society. 

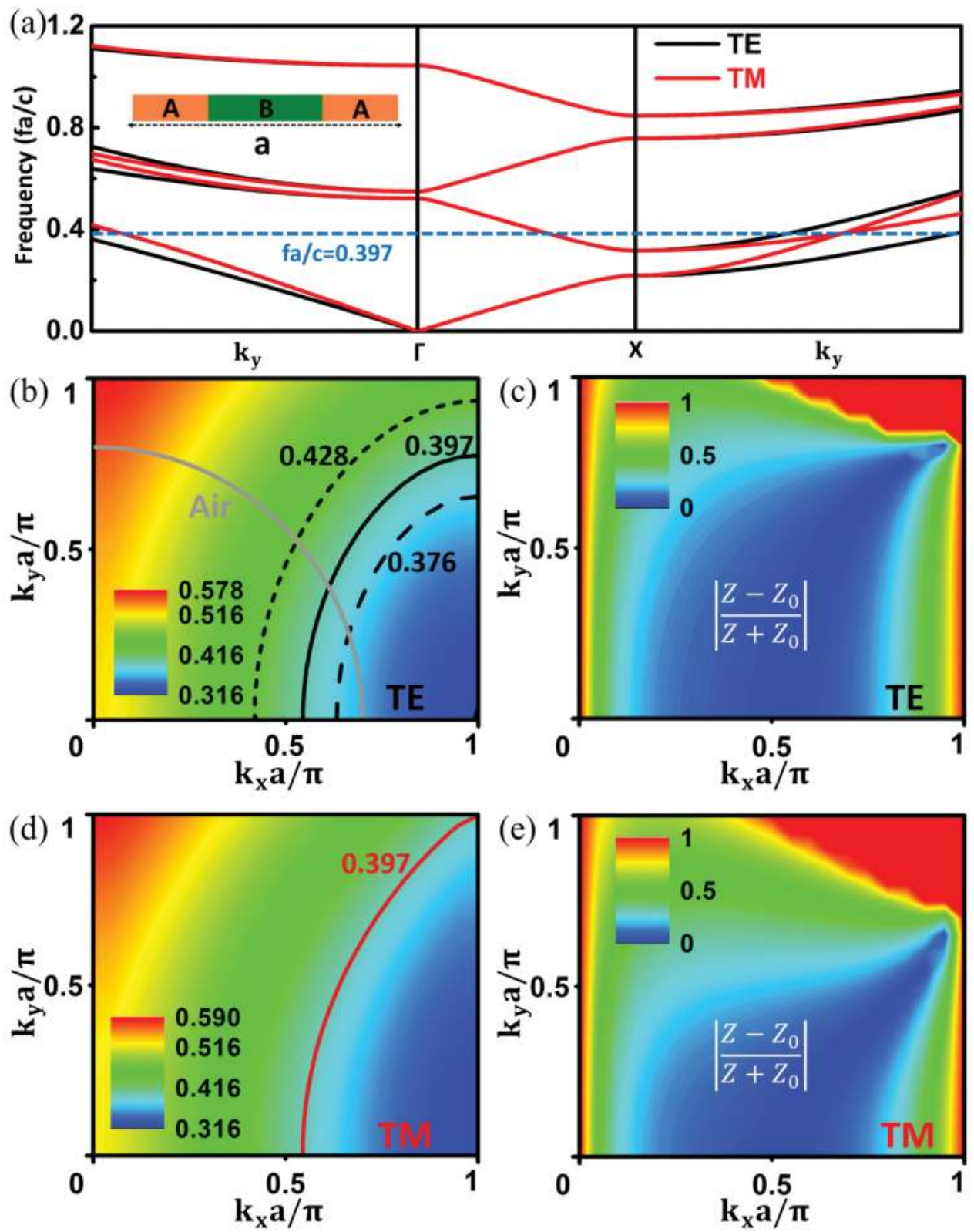

Figure 12. (a) Band structures of the PhC for TE and TM polarizations. The dashed lines denote the working frequency $f a / c=0.397$. The inset denotes the illustration of the unit cell. (b, d) the EFCs of the PhC and (c, e) the impedance difference between $\mathrm{PhC}$ and free space in the second band for (b, c) TE and (d, e) TM polarizations. Reprinted (figure) with permission from Ref. [22]. Copyright (2016) by the Optical Society. 
reciprocal space for both polarizations, demonstrating a wide-angle and polarizationinsensitive impedance matching effect. Moreover, from Figure 12(b) and (c), it is also seen that the impedance difference is small in the frequency range from $f a / c=0.376$ to $f a / c=0.428$, indicating a broadband impedance matching effect. Therefore, a one-dimensional PhC exhibiting a broadband, wide-angle, and polarization-insensitive impedance matching effect is realized.

For further verification, the transmittance through the PhC slab composed of $N$ unit cells is calculated. The numerical setup for the transmission computation is shown in Figure 13(a). A TE-polarized plane wave is incident from air in the left side. The upper and lower boundaries are set as periodic boundary condition. From the electric-field distribution in Figure 13(a), we see that almost all the waves can propagate through the PhC slab with 6 unit cells under the incident angle of $45^{\circ}$. In Figure 13(b), the transmittance is plotted as the functions of the incident angle and the number of unit cells $(N=4,5,6,15)$ for the frequency $f a / c=0.397$, showing $N$-independent almost perfect transmission for all incident angles of $\theta<70^{\circ}$.

Moreover, in Figure 14(a) and (b), the transmittance through a PhC slab $(N=10)$ as the functions of the incident angle and normalized frequency for TE and TM polarizations is presented, which clearly demonstrates the broadband, wide-angle, and polarization-insensitive transparency of the PhC.

Although a wide-angle $\left(0-70^{\circ}\right)$ rather than omnidirectional impedance matching effect is obtained in such a one-dimensional $\mathrm{PhC}$, we may still view such a $\mathrm{PhC}$ as an ultratransparent PhC. Compared with the two-dimensional ultratransparent PhCs, the one-dimensional ultratransparent PhCs have the advantages of broadband and polarization-insensitive impedance matching.
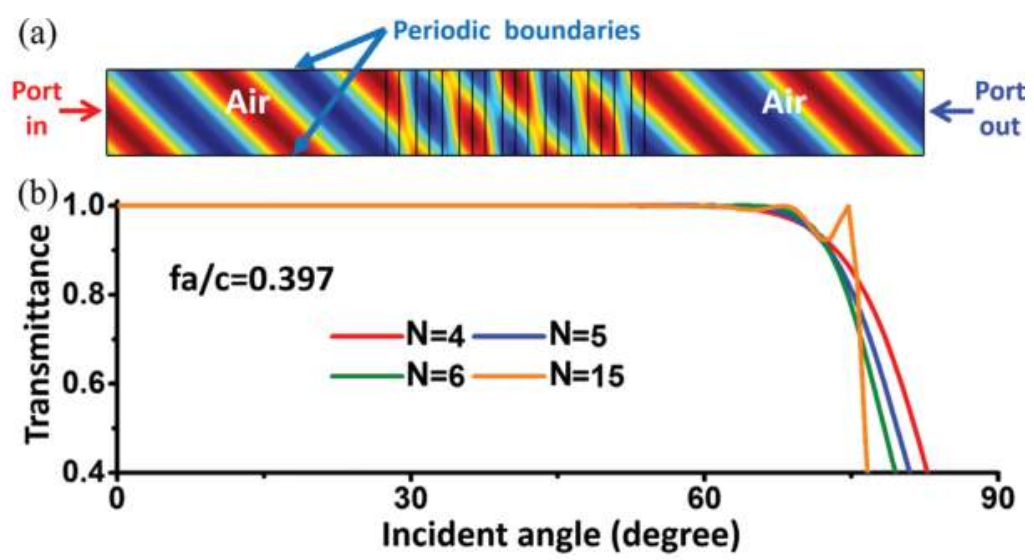

Figure 13. (a) The numerical setup for the transmission computation. The color denotes the distribution of electric fields under $\theta=45^{\circ}$ incident angle. (b) Transmittance as the function of the incident angle when EM waves propagate through the PhC slab composed of $N(=4,5,6,15)$ unit cells. Reprinted (figure) with permission from Ref. [22]. Copyright (2016) by the Optical Society. 

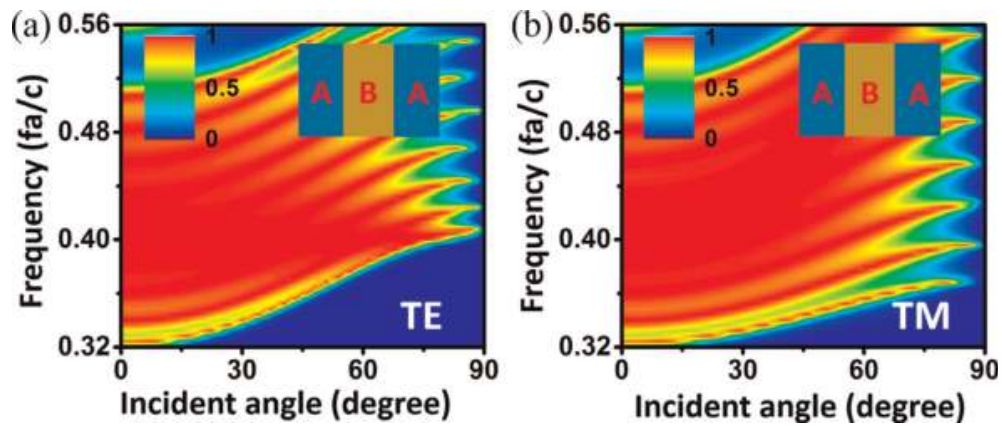

Figure 14. Transmittance as the functions of the incident angle and the frequency for (a) TE- and (b) TM-polarized waves propagating through the PhC slab with 10 unit cells. Reprinted (figure) with permission from Ref. [22]. Copyright (2016) by the Optical Society.

In addition, the easy design and fabrication of the one-dimensional PhCs may lead to more practical applications.

\section{Applications}

\subsection{For transformation optics}

In the above, we have demonstrated the ultratransparency in both one- and two-dimensional PhCs. In the following, we show some applications of such ultratransparent PhCs. It is interesting to note that the omnidirectional perfect transparency and elliptical EFCs of the ultratransparent media are essential for ideal TO devices. The theory of TO [23-27] promises many novel and interesting applications, such as invisibility cloaks [23, 25, 31, 32], concentrators [33], illusion optics devices [34-36], and simulations of cosmic phenomena [37, 38]. Generally, the TO devices are realized by using metamaterials [14-20], which require complicated designs of electric and magnetic resonances, hindering the realization and applications in practice. In fact, most of the previous TO experiments were realized by using the so-called reduced parameters, which maintain the refractive behavior, but sacrifice the impedance matching as well as the perfect transparency [25, 39-45]. Moreover, at optical frequencies, the inherent loss in metallic components of metamaterials makes the realization of perfect transparency as well as the ideal nonreflecting TO devices extremely difficult [46, 47], if not impossible. Interestingly, we find that the ultratransparent PhCs provide a low-loss and feasible platform for TO devices at optical frequencies.

To begin with, we consider a TO medium obtained by stretching the coordinate along the $x$ direction in background medium of air. For TE polarization with electric fields polarized in the $z$ direction, the parameters of the TO medium have the relationship [48]:

$$
\varepsilon_{z}=1 / \mu_{x}=\mu_{y}=1 / s
$$

where $s$ is the stretching ratio. $\varepsilon_{z}, \mu_{x}$ and $\mu_{y}$ are the $z$ component of relative permittivity tensor, $x$ and $y$ components of relative permeability tensor, respectively. 
Considering Eq. (12), the dispersion of the TO medium, i.e., $\frac{k_{x}^{2}}{\mu_{y}}+\frac{k_{y}^{2}}{\mu_{x}}=\varepsilon_{z} k_{0}^{2}$, can be rewritten as

$$
\frac{k_{x}^{2}}{1 / s^{2}}+k_{y}^{2}=k_{0}^{2}
$$

which has the similar form as that of Eq. (1). The EFC of the TO medium is an ellipse having the same height as the EFC of air in the $k_{y}$ direction (Figure 15(a)), as that of the ultratransparent media. When the stretching ratio is larger than unity, i.e., $s>1$, the EFC of the TO medium (the right ellipse) seems slimmer than the EFC of air (the left circle) in Figure 15(a), and the coordinate mesh is looser in the $x$ direction in the TO medium region, as illustrated in Figure 15(a).

The only difference of the EFCs between the TO medium and the ultratransparent medium is that there may exist a "shift" of $p$ in the $k_{x}$ direction (Eq. (1)) for the ultratransparent medium (Figure 15(b)). Interestingly, such a "shift" does not affect the refractive behaviors, but enables new freedom for phase manipulation beyond the local medium framework.

For demonstration, we show a specific example in Figure 16. The ultratransparent $\mathrm{PhC}$ is onedimensional and composed of components I and II. The unit cell is constructed in a symmetric way with a lattice constant of $a$, as illustrated by the inset in Figure 16(a). The relative permittivity and thickness of the component I (II) are 2.132 (5.522) and $0.3 a(0.4 a)$, respectively. In Figure 16(a), the EFC of the PhC at the normalized frequency $f a / c=0.402$ for TE polarization is plotted as the solid lines, which can be viewed as a part of an elliptical located at the $X$ point. Compared with the EFC of the TO medium with $\left\{\varepsilon_{z}, \mu_{x}, \mu_{y}\right\}=\{0.5,2,0.5\}$ (dashed lines in Figure 16(a)), we see that the EFC of the PhC has the same height in the $k_{y}$ direction, which satisfies the requirement proposed above.

Moreover, simulations of wave propagation through the TO medium slab with a thickness of $5 a$ (upper inset) and a PhC slab with 5 unit cells (lower inset) are performed, as shown in Figure 16(b).

(a)
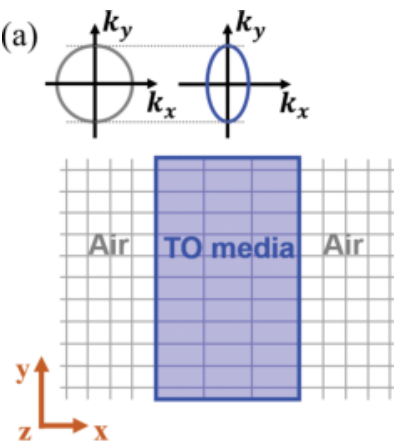

(b)
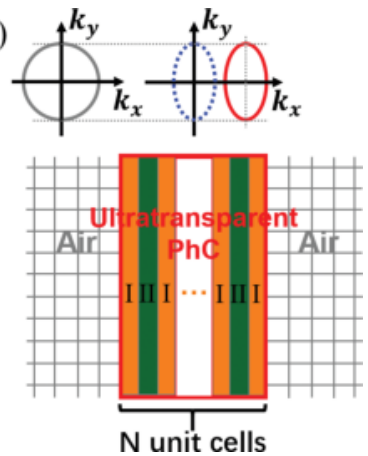

Figure 15. (a) Upper inset: the EFCs of air (the left circle) and TO media obtained by stretching air in the $x$ direction (the right ellipse). Lower inset: scheme of the TO medium slab (with loose mesh) in the background medium of air (with dense mesh). (b) Upper: the EFC of the ultratransparent PhC (the right ellipse with solid lines), which has a "shift" in the $k_{x}$ direction compared with the EFC of the TO media (dashed lines). Lower inset: the ultratransparent PhC is used to replace the TO medium slab in (a). 


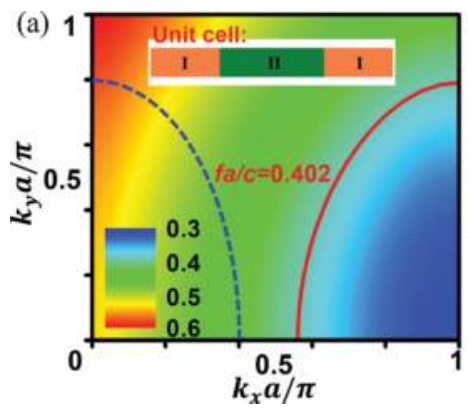

(b) 5 unit cells

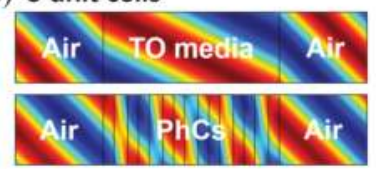

(c) 6 unit cells

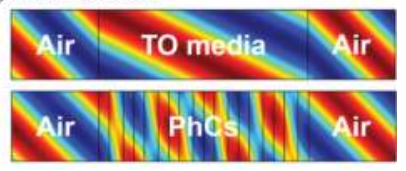

Figure 16. (a) The EFCs of the PhC for TE polarization. The solid line and dashed line denote the EFCs of the PhC and the TO medium at the normalized frequency $f a / c=0.402$, respectively. $(b, c)$ The snapshot of electric fields for wave propagating through a TO medium slab (upper inset) and a PhC slab (lower inset) under an incident angle of $45^{\circ}$. The thickness of the TO medium slab is (b) $5 a$, (c) $6 a$, and the number of unit cells of the PhC slab is (b) 5 , (c) 6 .

Simulation results show perfect transmission under the incident angle of $45^{\circ}$. Interestingly, we notice a $\pi$ phase difference in the transmission waves, which actually is caused by the "shift" of the PhC's EFC and odd number of unit cells. On the other hand, if the total number is even, there will be no phase difference, which is confirmed in the simulation results in Figure 16(c). In Figure 16(c), the thickness of the TO medium slab is $6 a$ (upper inset), and the number of unit cells of the PhC slab is 6 (lower inset). Comparing the transmission waves, we see identical phases under the incident angle of $45^{\circ}$. The results demonstrate that the ultratransparent $\mathrm{PhC}$ can approximately work as the TO medium with $\left\{\varepsilon_{z}, \mu_{x}, \mu_{y}\right\}=\{0.5,2,0.5\}$.

Next, we show an example of TO device by using one-dimensional ultratransparent PhCs. The design process is shown in Figure 17(a), in which the original shell of a concentrator [33] is discretized into four layers and each layer is further replaced by a corresponding ultratransparent PhC. Figure 17(b) shows the parameters of the discretized layers of TO media and the ideal profile. The corresponding four types of ultratransparent $\mathrm{PhCs}$ are of the same lattice constant $a$ and of 4, 2, 2, and 1 units for the A, B, C, and D layers, respectively. The EFCs of PhCs and the discretized layers of TO media are shown in Figure 17(c). It can be seen that the EFCs of PhCs have almost the same shapes with their corresponding layers, but are "shifted" by $\pi / a$ in the k-space.

The detailed parameters of the PhCs are presented in Figure 18. The insets present the illustrations of unit cells, relative permittivities, and thicknesses of each component of the four different PhCs. Moreover, the transmittance through PhC slabs with 10 unit cells is plotted as the function of incident angles, as shown by the solid lines in Figure 18(a-d). During the calculation, the background media are chosen as the discretized TO media (Figure 17(b)) with the parameters $\left\{\mu_{x}, \mu_{y}, \varepsilon_{z}\right\}$ being $\{1.52,0.658,0.793\},\{1.64,0.61,0.855\},\{1.84,0.543,0.96\}$, and $\{2.045,0.489,1.067\}$, respectively. It is clearly seen that the four PhCs all have near-unity transmittance in a wide-angle range $\left(0-70^{\circ}\right)$. 

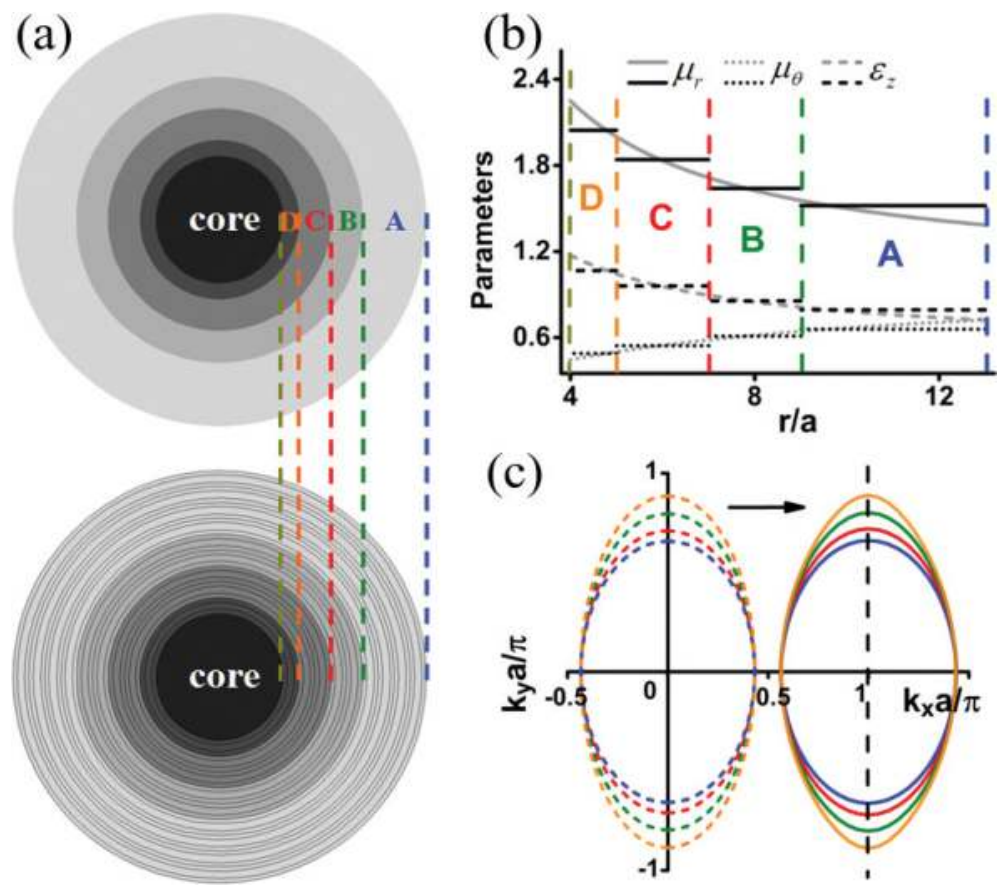

Figure 17. (a) Illustration of the design process from a concentrator composed of discretized layers of TO media (upper) to one composed of ultratransparent PhCs (lower). (b) Parameters of the discretized layers of TO media and the ideal profile. (c) EFCs of the discretized layers of TO media (dashed lines) and the corresponding PhCs (solid lines). Reprinted (figure) with permission from Ref. [21]. Copyright (2016) by the American Physical Society.

Moreover, numerical simulations are performed to demonstrate the functionality of the concentrator. Figure 19(a) and (b) corresponds to the concentrator composed of the original discretized TO media and the ultratransparent $\mathrm{PhCs}$, respectively. It is seen that under an incident beam of Gaussian wave from the lower left, both concentrators exhibit good concentration effects in the core areas and induce almost no scattering of waves. Interestingly, the waves inside the core areas exhibit a distinct phase difference of $\pi$. This discrepancy is a result of the new freedom introduced by the "shift" of EFCs in the k-space, i.e., spatial dispersion.

Therefore, we have demonstrated that ultratransparent media can work as the TO media to realize TO devices. Such ultratransparent media not only provide a low-loss and feasible platform for TO devices at optical frequencies, but also enable new freedom for phase manipulation beyond the local medium framework.

\subsection{For microwave transparency}

In the microwave regime, the ultratransparent media are also very useful and may have many applications in the design of radome, transparent wall, and so on. Here, we show an example 

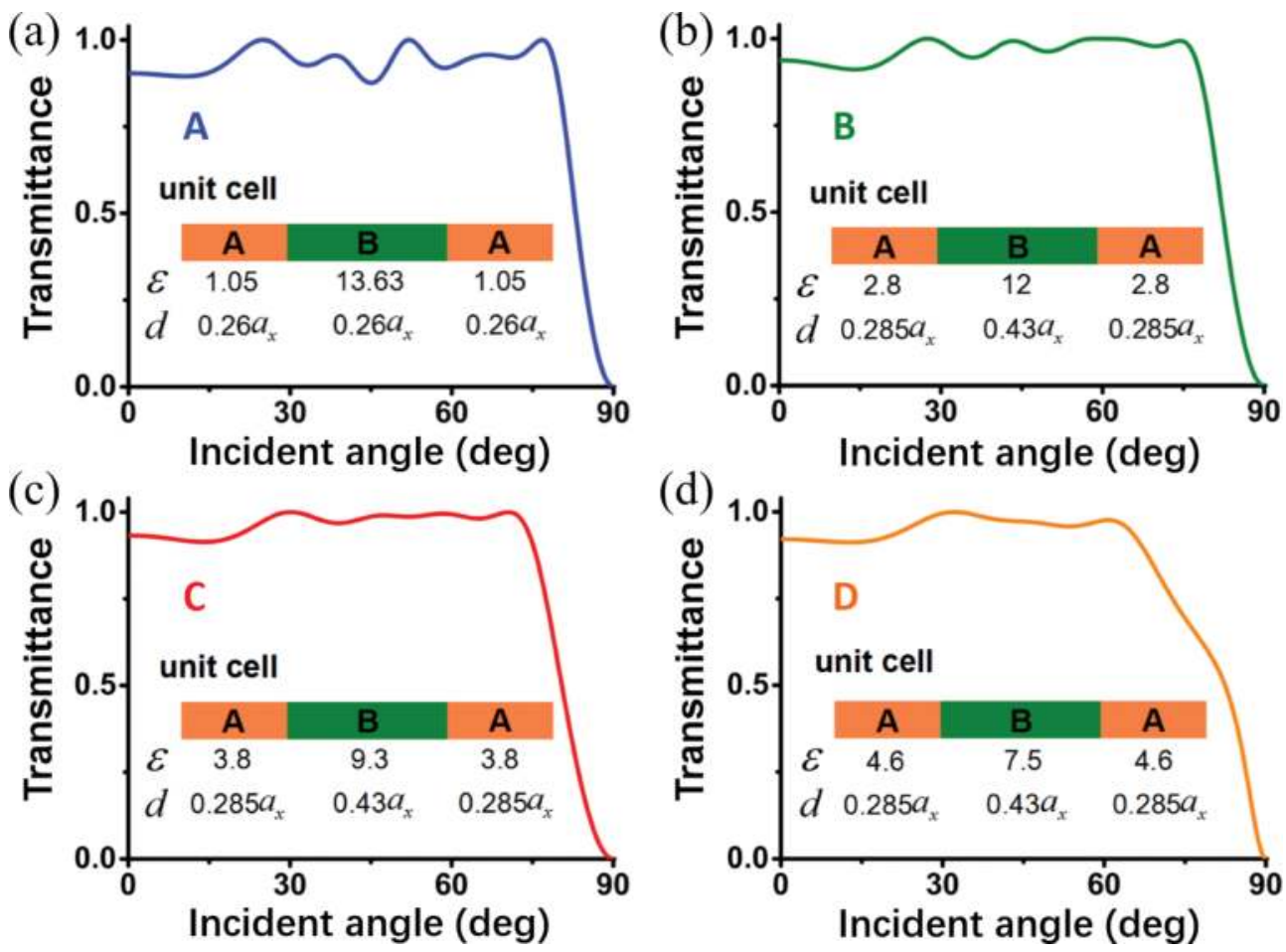

Figure 18. Transmittance through the PhC slabs with 10 unit cells as the function of incident angles. The PhCs in (a), (b), (c), and (d) correspond to the PhCs in regions A, B, C, and D, respectively. The insets are the structure, the relative permittivities, and the thicknesses of the components of the PhCs.
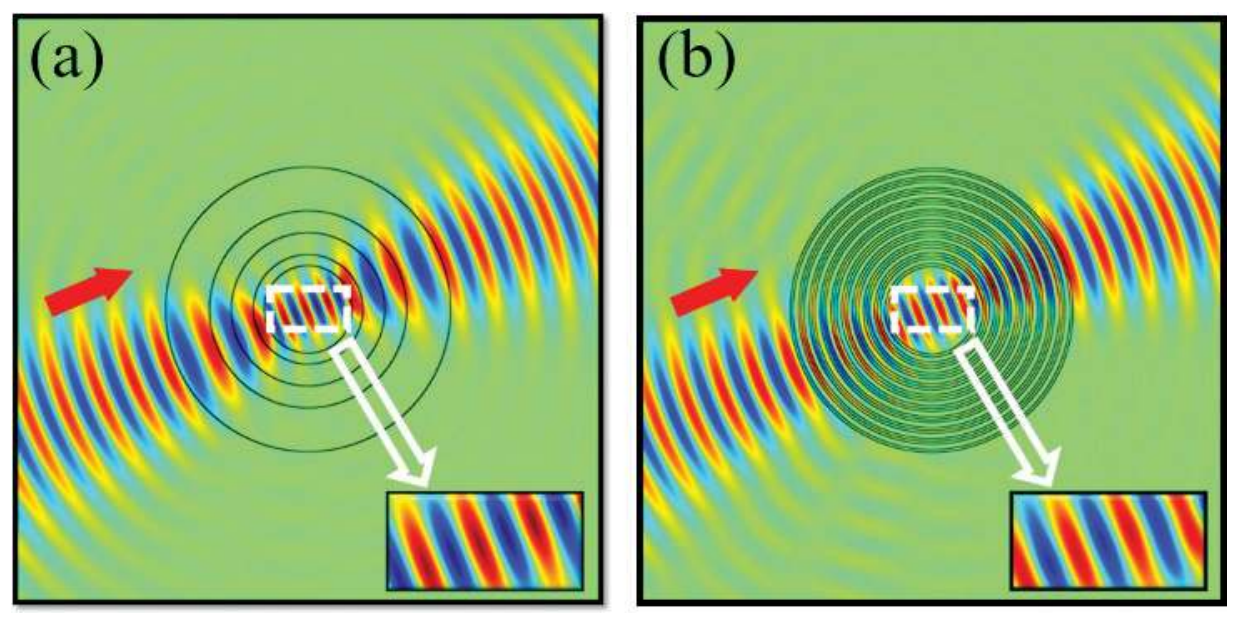

Figure 19. Electric field distributions in the concentrators made of (a) discretized TO media and (b) ultratransparent PhCs. Reprinted (figure) with permission from Ref. [21]. Copyright (2016) by the American Physical Society. 

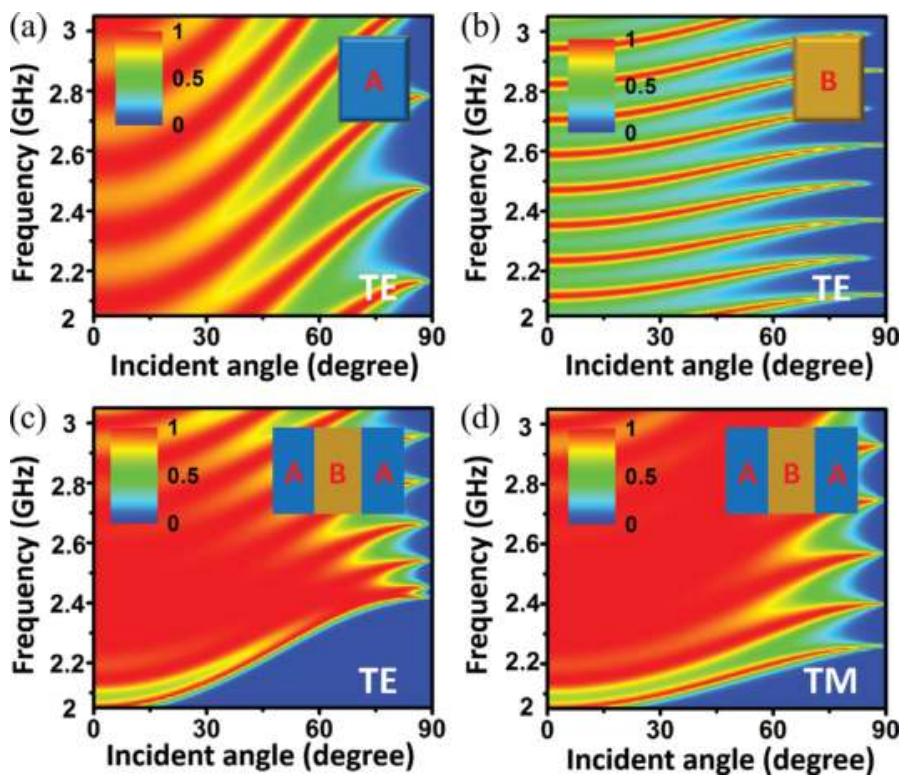

Figure 20. Transmittance with respect to the incident angle and the frequency for EM waves propagating through a slab made of (a) material A for the TE polarization, (b) material B for the TE polarization, (c, d) 10 unit cells of ABA for the (c) TE and (d) TM polarizations. Reprinted (figure) with permission from Ref. [22]. Copyright (2016) by the Optical Society.

of microwave transparent wall which allows the WiFi and $4 \mathrm{G}$ signals to pass through freely, and thus may find applications in architectural designs.

The microwave transparent wall is composed of one-dimensional ultratransparent $\mathrm{PhCs}$ with ABA unit cells. Materials $\mathrm{A}$ and $\mathrm{B}$ are chosen as polypropylene $\left(\varepsilon_{A}=2.3\right)$ and concrete $\left(\varepsilon_{B}=9\right.$ ). As we know, a wall (with a thickness of $42.5 \mathrm{~cm}$ ) composed of only material A or B would lead to Fabry-Pérot resonance-induced oscillation in the transmission spectrum, as shown by the Figure 20(a) and (b). Interestingly, if we utilize the composite structure with ABA unit cells, broadband, wide-angle, and polarization-insensitive transparency can be obtained. Specifically, the lattice constant $a$ of the unit cell is set to be $4.25 \mathrm{~cm}$, and the filling ratio of the material A (B) is $0.6(0.4)$. In Figure 20(c) and (d), the transmittance through a wall of 10 unit cells is plotted as the function of the incident angle and the working frequency for TE and TM polarizations, respectively. Obviously, almost $100 \%$ transmittance is obtained in a broad frequency band (from $2.3 \mathrm{GHz}$ to $2.7 \mathrm{GHz}$ ) and a wide-angle range (for almost all $\theta<60^{\circ}$ ) for both polarizations. We note that the frequency range covers the working frequency of Wi-Fi signal (with $2.4 \mathrm{GHz}$ ) and $4 \mathrm{G}$ signal $(2.3 \mathrm{GHz} 2.7 \mathrm{GHz}$ ). Thus, such microwave transparent walls may have applications in architectural designs.

\section{Conclusions and outlook}

In this chapter, we introduced the recent results of the structure-induced ultratransparency effect in both one- and two-dimensional PhCs, which allow near 100\% transmission of light 
for all incident angles and create aberration-free virtual images. The ultratransparency effect is well explained by nonlocal effective medium theory for PhCs and verified by both simulations and proof-of-principle microwave experiments. The design principle lies in systematic tuning of the microstructures of the PhCs based on the retrieved nonlocal effective parameters.

With the ultratransparent media, many applications can be expected such as the perfectly transparent optical lens, ideal TO devices, microwave transparent devices, and solar cell packaging. Interestingly, the ultratransparent media with "shifted" elliptical EFC not only provides a low-loss and feasible platform for TO devices at optical frequencies, but also enables new degrees of freedoms for phase manipulation beyond the local medium framework. In addition, microwave transparent walls allowing the WiFi and $4 \mathrm{G}$ signals to pass through freely can also be realized.

Although the ultratransparency effect is mainly demonstrated for TE polarization here, the principle is general and can be extended to TM polarization, or even both polarizations. Polarization-independent ultratransparency has wide and important applications. On the other hand, polarization-dependent ultratransparent media could also have some special applications. For instance, if the $\mathrm{PhC}$ is ultratransparent for TE polarization, while the working frequency falls in an omnidirectional band gap for TM polarization, such a PhC would work as an omnidirectional polarizer.

The concept and theory of ultratransparency give a guideline for pursuing solid materials with the ultimate transparency, i.e., broadband, omnidirectional, and polarization-insensitive total transparency. In the future, ultratransparent solid materials may be optimized to exhibit an unprecedented level of transparency and find vital applications in various fields.

\section{Acknowledgements}

This chapter is supported by National Natural Science Foundation of China (No. 11374224, 11574226, 11704271), Natural Science Foundation of Jiangsu Province (No. BK20170326), Natural Science Foundation for Colleges and Universities in Jiangsu Province of China (No. 17KJB140019), Jiangsu Planned Projects for Postdoctoral Research Funds (1701181B) and a Project Funded by the Priority Academic Program Development of Jiangsu Higher Education Institutions (PAPD).

\section{Author details}

Jie Luo and Yun Lai*

*Address all correspondence to: laiyun@suda.edu.cn

College of Physics, Optoelectronics and Energy and Collaborative Innovation Center of Suzhou Nano Science and Technology, Soochow University, Suzhou, China 


\section{References}

[1] Yablonovitch E. Inhibited spontaneous emission in solid-state physics and electronics. Physical Review Letters. 1987;58:2059-2062

[2] John S. Strong localization of photons in certain disordered dielectric superlattices. Physical Review Letters. 1987;58:2486-2489

[3] Joannopoulos JD, Villeneuve PR, Fan S. Photonic crystals: Putting a new twist on light. Nature. 1997;386:143-149

[4] Joannopoulos JD, Johnson SG, Winn JN, Meade RD. Photonic Crystals: Molding the Flow of Light. 2nd ed. Princeton, USA: Princeton University Press; 2008:63-121

[5] Sakoda K. Optical Properties of Photonic Crystals. New York, USA: Springer; 2005:99-250

[6] Ho K, Chan C, Soukoulis C. Existence of a photonic gap in periodic dielectric structures. Physical Review Letters. 1990;65:3152-3155

[7] Fink Y, Winn JN, Fan S, Chen C, Michel J, Joannopoulos JD, Thomas EL. A dielectric omnidirectional reflector. Science. 1998;282:1679-1682

[8] Mekis A, Chen JC, Kurland I, Fan SH, Villeneuve PR, Joannopoulos JD. High transmission through sharp bends in photonic crystal waveguides. Physical Review Letters. 1996;77: 3787-3790

[9] Lin S, Chow E, Hietala V, Villeneuve PR, Joannopoulos JD. Experimental demonstration of guiding and bending of electromagnetic waves in a photonic crystal. Science. 1998;282: 274-276

[10] Russell P. Photonic crystal fibers. Science. 2003;299:358-362

[11] Painter O, Lee RK, Scherer A, Yariv A, O'Brien JD, Dapkus IKPD. Two-dimensional photonic band-gap defect mode laser. Science. 1999;284:1819-1821

[12] Yamamoto T, Pashkin YA, Astafiev O, Nakamura Y, Tsai JS. High-Q photonic nanocavity in a two-dimensional photonic crystal. Nature. 2003;425:941-944

[13] Shen Y, Ye D, Celanovic I, Johnson SG, Joannopoulos JD, Soljacic M. Optical broadband angular selectivity. Science. 2014;343:1499-1501

[14] Pendry JB, Holden AJ, Stewart WJ, Youngs II. Extremely low frequency plasmons in metallic mesostructures. Physical Review Letters. 1996;76:4773-4776

[15] Pendry JB, Holden AJ, Robbins DJ, Stewart WJ. Magnetism from conductors and enhanced nonlinear phenomena. IEEE Transactions on Microwave Theory and Techniques. 1999;47: 2075-2084

[16] Smith DR, Padilla WJ, Vier DC, Nemat-Nasser SC, Schultz S. Composite medium with simultaneously negative permeability and permittivity. Physical Review Letters. 2000;84: $4184-4187$ 
[17] Shelby RA, Smith DR, Schultz S. Experimental verification of a negative index of refraction. Science. 2001;292:77-79

[18] Pendry JB. Negative refraction makes a perfect lens. Physical Review Letters. 2000;85: 3966-3969

[19] Zheludev NI. The road ahead for metamaterials. Science. 2010;328:582-583

[20] Liu Y, Zhang X. Metamaterials: A new frontier of science and technology. Chemical Society Reviews. 2011;40:2494-2507

[21] Luo J, Yang Y, Yao Z, Lu W, Hou B, Hang ZH, Chan CT, Lai Y. Ultratransparent media and transformation optics with shifted spatial dispersions. Physical Review Letters. 2016;117:223901

[22] Yao Z, Luo J, Lai Y. Photonic crystals with broadband, wide-angle, and polarizationinsensitive transparency. Optics Letters. 2016;41:5106-5109

[23] Pendry JB, Schurig D, Smith DR. Controlling electromagnetic fields. Science. 2006;312: 1780-1782

[24] Leonhardt U. Optical conformal mapping. Science. 2006;312:1777-1780

[25] Schurig D, Mock JJ, Justice BJ, Cummer SA, Pendry JB, Starr AF, Smith DR. Metamaterial electromagnetic cloak at microwave frequencies. Science. 2006;314:977-980

[26] Chen H, Chan CT, Sheng P. Transformation optics and metamaterials. Nature Materials. 2010;9:387-396

[27] Pendry JB, Aubry A, Smith DR, Maier SA. Transformation optics and subwavelength control of light. Science. 2012;337:549-552

[28] Śmigaj W, Gralak B. Validity of the effective-medium approximation of photonic crystals. Physical Review B. 2008;77:235445

[29] Lu Z, Prather DW. Calculation of effective permittivity, permeability, and surface impedance of negative-refraction photonic crystals. Optics Express. 2007;15:8340-8345

[30] Lawrence FJ, de Sterke CM, Botten LC, McPhedran RC, Dossou KB. Modeling photonic crystal interfaces and stacks: Impedance-based approaches. Advances in Optics and Photonics. 2013;5:385

[31] Li J, Pendry JB. Hiding under the carpet: A new strategy for cloaking. Physical Review Letters. 2008;101:203901

[32] Lai Y, Chen H, Zhang Z, Chan C. Complementary media invisibility cloak that cloaks objects at a distance outside the cloaking shell. Physical Review Letters. 2009;102:093901

[33] Rahm M, Schurig D, Roberts DA, Cummer SA, Smith DR, Pendry JB. Design of electromagnetic cloaks and concentrators using form-invariant coordinate transformations of Maxwell's equations. Photonics and Nanostructures. 2008;6:87-95 
[34] Lai Y, Ng J, Chen H, Han D, Xiao J, Zhang Z, Chan C. Illusion optics: The optical transformation of an object into another object. Physical Review Letters. 2009;102:253902

[35] Pendry J. All smoke and metamaterials. Nature. 2009;460:79-580

[36] Lai Y, Ng J, Chen H, Zhang Z, Chan CT. Illusion optics. Frontiers of Physics China. 2010;5:308-318

[37] Genov DA, Zhang S, Zhang X. Mimicking celestial mechanics in metamaterials. Nature Physics. 2009;5:687-692

[38] Sheng C, Liu H, Wang Y, Zhu SN, Genov DA. Trapping light by mimicking gravitational lensing. Nature Photonics. 2013;7:902-906

[39] Cai W, Chettiar UK, Kildishev AV, Shalaev VM. Optical cloaking with metamaterials. Nature Photonics. 2007;1:224-227

[40] Liu R, Ji C, Mock JJ, Chin JY, Cui TJ, Smith DR. Broadband ground-plane cloak. Science. 2009;323:366-369

[41] Valentine J, Li J, Zentgraf T, Bartal G, Zhang X. An optical cloak made of dielectrics. Nature Materials. 2009;8:568-571

[42] Gabrielli LH, Cardenas J, Poitras CB, Lipson M. Silicon nanostructure cloak operating at optical frequencies. Nature Photonics. 2009;3:461-463

[43] Ergin T, Stenger N, Brenner P, Pendry JB, Wegener M. Three-dimensional invisibility cloak at optical wavelengths. Science. 2010;328:337-339

[44] Ma HF, Cui TJ. Three-dimensional broadband ground-plane cloak made of metamaterials. Nature Communications. 2010;1:21

[45] Xu S, Cheng X, Xi S, Zhang R, Moser HO, Shen Z, Xu Y, Huang Z, Zhang X, Yu F, Zhang B, Chen $\mathrm{H}$. Experimental demonstration of a free-space cylindrical cloak without superluminal propagation. Physical Review Letters. 2012;109:223903

[46] Xiao S, Drachev VP, Kildishev AV, Ni X, Chettiar UK, Yuan HK, Shalaev VM. Loss-free and active optical negative-index metamaterials. Nature. 2010;466:735-738

[47] Hess O, Pendry JB, Maier SA, Oulton RF, Hamm JM, Tsakmakidis KL. Active nanoplasmonic metamaterials. Nature Materials. 2012;11:573-584

[48] Lai Y, Zheng H, Zhang Z, Chan CT. Manipulating sources using transformation optics with 'folded geometry'. Journal of Optics. 2011;13:024009 
